\title{
Sí, profe, pero... La enseñanza de la argumentación oral en el aula de ELE
}

\author{
Amparo Alemany MartíneZ \\ Universitat de València \\ amparo.alemany@uv.es
}

\begin{abstract}
Resumen: El trabajo que se presenta es una propuesta teórico-práctica sobre la argumentación oral en el aula de ELE a partir de ciertos conceptos argumentativos como los topoi o los tipos de argumentos. El estudio se divide en dos secciones: por una parte, se mostrarán las bases teóricas en las que se sustenta la propuesta y; por otra parte, se propondrá una serie de actividades para llevar a cabo en el aula de ELE con el objetivo de mejorar las habilidades argumentativas de nuestro alumnado.
\end{abstract}

Palabras clave: argumentación en la lengua, topoi, orientación argumentativa.

Yes, teacher, but... Learning to teach oral argumentation in ELE classroom

Abstract: The aim of this paper is to explain how the theory of arguments can help ELE students to improve their oral argumentative skills. For instance, notion like topoi, which is a concept of the theory of arguments based on Anscombre and Ducrot approach, or even the types of arguments. To do this, this paper first displays the theoretical basis of this framework, and second it promotes a set of activities to achieve an improvement in the student's argumentative skills.

Key words: theory of arguments, topoi, argumentative orientation

\section{La argumentación en ELE: ¿Por qué se debe enseñar argumentación oral?}

En la actualidad, se observa una creciente tendencia de personas que carecen de la competencia para argumentar apropiadamente; hecho que se demuestra, por ejemplo, con un aumento del impacto de las fake news. Esto sucede también con los alumnos de enseñanzas obligatorias (Dolz y Pasquier, 1996: 95) e incluso postobligatorias (Álvarez y García, 2016: 19). En relación con el aula de ELE, la argumentación se viene estudiando a partir de niveles superiores, como el B2:

disponen de capacidad lingüística suficiente como para plantear los detalles de un problema, presentar reclamaciones y resolver situaciones conflictivas recurriendo a su capacidad de argumentar y a un lenguaje persuasivo (Plan Curricular del Instituto Cervantes, PCIC, 2006) ${ }^{1}$.

Entendemos, por tanto, que argumentar sobre un tema lleva consigo cierta intencionalidad: convencer, persuadir, expresarse o simplemente ratificarse como hablante (Camps y Dolz, 1995: 5). Consideramos, del mismo modo, que argumentar forma parte del «uso ordinario de la lengua» (Plantin, 1998: 24 en Cuenca, 1995: 29) y, debido a ello, entendemos que lo correcto para reflejar una opinión es fundamentarla y, por tanto, argumentarla.

\footnotetext{
${ }^{1}$ El subrayado es nuestro.
} 
Por tanto, dado que la argumentación es una estrategia de carácter diario y que se contempla en el currículum del Instituto Cervantes y del Marco Común Europeo de Referencia para las Lenguas (MCERL), el objetivo de este trabajo consistirá en proponer un conjunto de actividades que ayuden a los estudiantes de ELE a desarrollar su competencia argumentativa oral $\mathrm{y}$, de esta forma, sean capaces de defender sus opiniones e, incluso, formar parte de cualquier conversación que implique un mínimo de debate. Para ello, este estudio da una serie de herramientas tanto teóricas $(\S 2)$ como prácticas $(\S 3)$ para realizar una secuencia didáctica $(\S 4)$, aplicada a estudiantes de un nivel B2 o superior para que el alumnado no nativo en español se familiarice con la argumentación oral.

Cabe decir que este artículo tiene una limitación de base y, es que las actividades no han sido realizadas en un entorno de ELE, sino que se han llevado a cabo en secundaria (en concreto, en el nivel de $1^{\circ}$ de bachiller). Sin embargo, dados los resultados positivos de esta implementación, se ha querido desplazar lo conseguido al aula de ELE para tratar de crear una secuencia didáctica que permita a nuestros alumnos no nativos desarrollar su competencia argumentativa en la lengua oral.

\section{Algunas bases teóricas}

La argumentación es una estrategia propia de los seres humanos (Cuenca, 1995: 24) que, dependiendo de la vertiente teórica que tomemos, puede divergir significativamente (Plantin, 1998: 13-21; Plantin, 2012: 19-45; Escandell, 2013: 94, entre otros). A grandes rasgos, como actividad discursiva existen dos formas de llevarla a cabo (Rodríguez Gonzalo, 2012: 8-10): en primer lugar, de manera científica, donde la validez de los argumentos se consigue por medio del método científico y la asunción de datos empíricamente demostrables y, en segundo lugar, desde una perspectiva social, donde normalmente existe una finalidad persuasiva (Anscombre y Ducrot, 1994) y un componente de opinión:

Mais on parle souvent en cherchant à faire partager à un interlocuteur des opinions ou des représentations relatives à un thème donné, en cherchant à provoquer ou accroitre I'adhésion d'un auditeur ou d'un auditoire plus vaste aux thèses qu'on présente à son assentiment. (Adam, 1992: $105)^{2}$

Pensamos que ambas líneas teóricas no se contraponen, sino que en mayor o menor medida se complementan y son necesarias para la formación de la competencia argumentativa del alumnado. No obstante, para este trabajo nos acogeremos a la perspectiva más social, dado que se relaciona ampliamente con el contenido que tanto el Plan Curricular del Instituto Cervantes como el Marco Común Europeo describen para el nivel B2 y superiores:

«Can evaluate the position of one party in a disagreement and invite them to reconsider an issue, relating his/her argumentation to that party's stated aim» (MCERL, 2018: apéndice 9)

\footnotetext{
${ }^{2}$ El subrayado es nuestro.
} 


\subsection{La argumentación de la lengua y en la lengua}

En este punto, resumiremos las principales ideas sobre la historia de la argumentación y los presupuestos de partida que hacen que sea un fenómeno interesante para el estudio desde distintas dimensiones teóricas aplicadas al contexto del aula.

Plantin (2012: 20-23) establece que tradicionalmente la argumentación se puede estudiar desde distintas perspectivas: la retórica (la técnica de persuadir por medio del lenguaje a un público) la dialéctica, (una perspectiva dialogal entre un oponente y un proponente) y; finalmente, la lógica, (encargada del estudio de la conclusión a la que se llega a través de las premisas que se aportan). Las tres disciplinas poseen puntos en contacto $\mathrm{y}$, además, la retórica y la dialéctica funcionan como dos caras de la misma moneda, aunque con consideraciones distintas (Van Eemeren y Grootendorst, 1992; Zamudio, 2012) ${ }^{3}$.

Todas las perspectivas anteriores podrían considerarse en una teoría sobre la argumentación de la lengua; es decir, estas entienden la argumentación como una manera de utilizar la lengua en ciertas situaciones dadas, ya sea con fines lógicos, dialécticos o retóricos. Sin embargo, existe otra corriente que entiende que la argumentación es inherente a la propia lengua y se encuentra dentro de ella en un sentido literal.

A diferencia de las teorías anteriores, cuando hablamos de argumentación en la lengua, nos referimos a una concepción que se basa en «presentar un enunciado A como si tuviera que llevar al destinatario a concluir una conclusión $\mathrm{C}$ » o en «dar A como una razón para creer C» (Anscombre y Ducrot, 1994: 36). Dicho de otro modo, mientras que otras visiones ponen el foco en la lógica o en la recepción del discurso, autores como Anscombre y Ducrot analizan el hecho argumentativo de forma puramente lingüística.

Uno de los motivos que nos ha llevado a la realización de este trabajo es su consideración del topos como garante argumentativo (Anscombre y Ducrot, 1994: 217). Este concepto empezó siendo una categoría aristotélica definida como un enunciado «general que expresa un principio susceptible de engendrar una infinidad de argumentaciones concretas y particulares» (Plantin, 2012: 35). No obstante, a partir de la Teoría de la Argumentación en la lengua (Anscombre y Ducrot, 1994) se empezó a considerar el garante de coherencia entre la unión de dos encadenamientos argumentativos (Anscombre y Ducrot, 1994: 217):

El topos, relación argumentativa o ley de paso, es la conexión entre dos hechos o conceptos, que la comunidad ha socializado y que permite la relación entre los argumentos para llegar a una conclusión (Fuentes y Alcaide, 2007: 36).

Esto guarda relación con la ordenación de los argumentos, ya que en la lengua el orden de los factores sí que altera el producto y el encadenamiento de las razones que sustentan nuestra tesis pueden ser efectivas dependiendo del lugar que ocupen en nuestro discurso, un ejemplo de ello:

\footnotetext{
${ }^{3}$ En este párrafo se ha pretendido realizar una síntesis de las numerosas propuestas sobre la argumentación existentes. No se ha pretendido en ningún caso un grado excesivo de profundidad. Si el lector quisiera consultar más información sobre argumentación, podría remitirse a los siguientes autores: retórica (Perelman y Olbrechtstyteca, 1958; Cattani, 2003, Abascal, 2005, etc.), dialéctica (Schopenhauer, 1864; Van Eemeren y Grootendorst, 2004; Zamudio, 2012; Johnson, 2013; etc.), lógica (Toulmin, 1958, Fischer, 2004; etc.).
} 
Un jesuita presumía delante de un dominico, y con un cigarrillo en la mano, de que a ellos sus superiores les permitían fumar mientras rezaban. El dominico comentaba que, por el contrario, a ellos se lo habían prohibido. El jesuita le preguntó cómo habían planteado la petición a su padre superior. Y el dominico le respondió que simplemente habían preguntado si podían fumar mientras rezaban. El jesuita le dijo entonces: «no es ésa la mejor forma de conseguir el permiso. Nosotros le preguntamos al padre superior si podíamos rezar mientras fumábamos.» ${ }^{4}$ (Grijelmo, 2012: 34).

Los topoi, además, de manera estructural, se definen como afines a una comunidad lingüística dada (a); es decir, todos los interlocutores de la misma los entienden, lo cual hace que estos también sean generales (b), ya que pueden valer para varias situaciones y; además son graduales (c) (Anscombre y Ducrot, 1994: 218). Valga el ejemplo que muestra Escandell (2013: 107) para definir topos bajo estas tres premisas básicas:

(1) María sabe mucho: tiene una licenciatura y ha terminado el doctorado

Esto es, la relación tópica existente en (1) vincula dos conceptos: por un lado, el grado de instrucción educativa (licenciada y doctorada) y; por otro, la sabiduría que le otorgan esos estudios a María. Por lo tanto, el topos formulado tiene que ver con la concepción de nuestra comunidad por lo que entendemos por sabiduría, es decir, en este caso tener un doctorado y; debido a ello, el topos sería: a más instrucción educativa, más sabiduría.

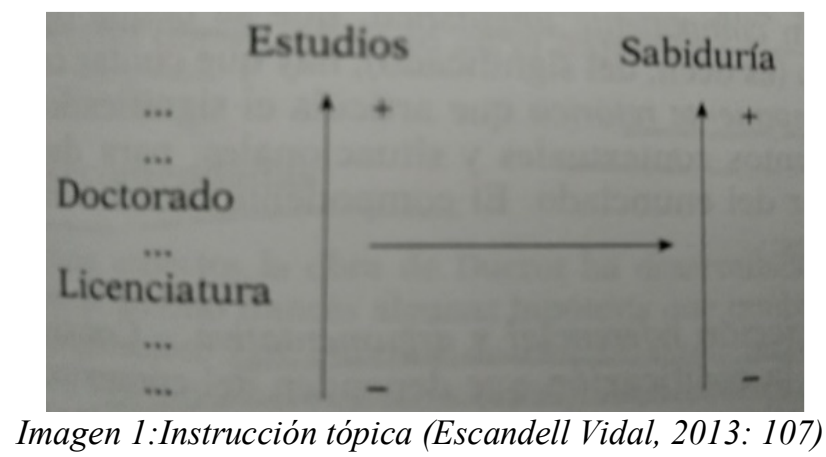

En conclusión, creemos que estas perspectivas teóricas resultan útiles para la enseñanza de la argumentación en tanto que fundamentan las explicaciones discursivas, sea a través de un esquema organizativo previo (Toulmin, 1958), mediante una retórica que busca convencer de manera universal a un interlocutor con mecanismos dialécticos (Perelman y Olbrechts-Tyteca, 1958) o mediante la consideración de que la lengua per se tiene intención argumentativa y este hecho es inmanente a la comunicación tanto oral como escrita (Anscombre y Ducrot, 1994).

\subsection{Argumentar la opinión personal: ¿es objetiva la argumentación?}

La argumentación no siempre se caracteriza por ser totalmente objetiva con los hechos, muchas veces tiene un fuerte componente de opinión personal y, además, este hecho guía el contenido de los razonamientos que se justifican. Por este motivo, debemos de aspirar a ser convincentes y lo suficientemente críticos con la postura en contra como para convencer a un interlocutor o varios (Felton, 2007). Sin embargo, en algunas ocasiones los estudiantes no logran argumentar con fundamento:

\footnotetext{
${ }^{4}$ El subrayado es nuestro.
} 
Un argumento no es simplemente la afirmación de ciertas opiniones, ni se trata simplemente de una disputa. Los argumentos son intentos de apoyar ciertas opiniones con razones [...]. Muchos estudiantes, $[\ldots]$ transcriben elaboradas afirmaciones de sus opiniones, pero no ofrecen ninguna auténtica razón para pensar que sus propias opiniones son las correctas. Escriben un ensayo, pero no un argumento. (Weston, 2006: 11-13)

$\mathrm{O}$, dicho de otra manera, la opinión personal es fundamental cuando se construye una argumentación; sin embargo, debe estar bien razonada para que pueda convencer y persuadir. Por esta misma razón, hay que conocer las opiniones contrarias a la propia, para evitar en primer lugar el sesgo de confirmación y, en segundo lugar, para construir mejores argumentaciones basadas en datos (Jiménez Aleixandre, 2009: 173).

No obstante, puede parecer idílico que esto resulte provechoso en el aula de ELE ya que, a diferencia de un alumnado nativo; posiblemente nuestros alumnos extranjeros no tengan las herramientas lingüísticas necesarias para lidiar con la información que justifica sus opiniones y; al mismo tiempo, con la que las desacreditan. Por tanto, nuestra tarea a partir de las actividades prácticas que se propondrán a continuación $(\S 4)$ es conseguir que nuestros aprendientes sepan justificarse y dar su opinión de una manera eficiente y coherente con lo marcado a partir de su nivel de instrucción lingüística; esto es, B2 (o superior).

Credo importante che i ragazzi abbiano occasione di sperimentare nella scuola anche il campo delle opinioni, per sviluppare un atteggiamento immune dal dogmatismo e per avere l'esperienza della responsabilità personale delle proprie affermazioni. (Colombo, 1992: 60)

\subsection{En el aula, ¿cómo se trata la argumentación?}

En este apartado reseñaremos brevemente lo que dice el marco legal actual acerca de la argumentación principalmente en ELE. En concreto, enseñar español tiene como principal objetivo, independientemente del nivel educativo que se trate, desarrollar en el alumnado la competencia comunicativa en su totalidad, esto es, conseguir un correcto aprendizaje de contenidos pragmáticos, lingüísticos, sociolingüísticos y literarios para cualquier contexto que se presente (RD 1105/2014: 357; Decreto 87/2015: 1).

Se ha optado por establecer la propuesta en un nivel B2 o superior, debido a que uno de los principales objetivos de este nivel (en relación con la lengua oral) según el renovado MCERL son:

Can with some effort catch much of what is said around him/her, but may find it difficult to participate effectively in discussion with several speakers of the target language who do not modify their speech in any way. Can identify the main reasons for and against an argument or idea in a discussion conducted in clear standard speech. Can follow chronological sequence in extended informal speech, e.g. in a story or anecdote (MCERL, 2018: 56)

Como se puede apreciar, ya en los principales objetivos del B2 se muestra la identificación de los argumentos a favor y en contra en un debate. Esto es, la argumentación es un eje vertebrador para los aprendientes de español cuando interactúan con otros hablantes y, al mismo tiempo, la identificación de la orientación argumentativa de ciertos enunciados dados (topoi). La argumentación es un apartado importante para conseguir que nuestros aprendientes desarrollen adecuadamente una competencia comunicativa, se expresen con claridad y sin ambigüedad y; además consigan afianzar un pensamiento autónomo, crítico y reflexivo. 
También el PCIC (2006) en su apartado titulado macrofunción argumentativa propone al nivel B2 como el óptimo para aprender estrategias como la contraargumentación o la defensa de una tesis polémica en sus apartados. De hecho, advierte que los aprendientes de español son agentes sociales y, en este sentido:

Durante el transcurso de reuniones profesionales o seminarios, pueden seguir el ritmo de discusiones animadas e identifican los argumentos de los diferentes puntos de vista; expresan sus ideas y opiniones con precisión, presentan líneas argumentativas de cierta complejidad y responden a ellas con convicción; (PCIC, 2006).

En relación con esto, debido a que consideramos que la capacidad argumentativa es una de las bases que garantiza una correcta reflexión lingüística, entendemos que nuestra propuesta de actividades ha de ir encaminada a conseguir una serie de objetivos que promuevan; por un lado, la reflexión en nuestros aprendientes y, por otro, el aprendizaje de un tipo de argumentación que en vez de ganar o perder argumentando, convenza con la palabra.

La argumentación, por tanto, es necesaria para trabajar la competencia existencial o savoir-être («saber ser») en materia de actitudes $(P C I C, 2006)$ tolerantes con respecto a la escucha de argumentos que no compartimos mientras debatimos con algún hablante; con ello, por ejemplo, se aprende a escuchar otras opiniones que no tienen por qué ser la propia.

\section{Metodología}

El trabajo que se presenta, como ya veníamos diciendo, parte de una unidad didáctica implementada en el aula de secundaria, en concreto sobre la manera de construir encadenamientos argumentativos a partir de un tema dado con el alumnado de $1 .^{\circ}$ Bachillerato (16-17 años) de un Instituto de Enseñanza Secundaria (IES). No obstante, hemos adaptado esta propuesta al aula de ELE con el fin de reforzar y mejorar la capacidad argumentativa, crítica y reflexiva de los aprendientes de español (Reyzábal, 2002: 56) a partir de la base de los topoi. Por tanto, se mostrará la secuencia didáctica (SD) que se llevó a cabo en secundaria de forma íntegra (§ 3.1.) para, posteriormente, adecuarla en un contexto de enseñanza de español a extranjeros; en concreto, en un nivel B2 o superior (§ 3.2. y $\S 4$.).

\subsection{La secuencia didáctica en secundaria}

\subsubsection{Objetivos}

La SD que se presenta a continuación pretendía mejorar la competencia argumentativa del alumnado. La tarea final de esta secuencia era llevar a cabo un debate sobre los roles de género y los estereotipos sociales que se le asignan a cada sexo.

Por tanto, la SD como propuesta de intervención de aula en secundaria tuvo varios objetivos:

a) Conseguir que los estudiantes mejoren en la construcción de sus argumentaciones con el fin de expresar su opinión de manera más contrastada y rica, sustituyendo las categorías de ganar o perder por las de convencer y razonar. 
b) Ampliar su perspectiva lingüística mediante la crítica argumentativa a partir del uso de argumentos que convenzan a sus interlocutores (tanto lectores como oyentes).

Para conseguir estos objetivos se realizó un diseño de SD que tenía en cuenta una serie de premisas previas:

a) Los alumnos de Bachillerato en la práctica no distinguen argumentativamente debate de discusión.

b) Las argumentaciones del estudiantado de Secundaria, tanto en el oral como en el escrito, tienden a ser falaces, debido a que los estudiantes necesitan tener antes la razón que argumentar con propiedad.

A partir de las premisas anteriores, se diseñaron ciertas clases y tareas que permitieran al alumnado asimilar y trabajar la competencia argumentativa adecuadas al nivel de $1^{\circ}$ de Bachillerato.

\subsubsection{Diseño de la intervención}

El diseño de la intervención se llevó a cabo mediante una serie de referencias sobre el aprovechamiento de la argumentación de la opinión personal (Dolz y Pasquier, 1995, 2000; Sánchez Prieto, 2007, entre otros); y, sobre todo a través de propuestas didácticas del debate como las del Grupo Gorgias (2013) o de la persuasión (Reyzábal, 2002). Para ello, se diseñaron las siguientes sesiones:

Número de sesiones

1. ${ }^{\circ}$ Sesión: toma de contacto con la argumentación

2. ${ }^{\circ}$ Sesión: ¿debate? ¿qué es debatir?

\section{Sesión: preparación para} el debate final

\section{4. ${ }^{\circ}$ Sesión: debate final y} puesta en común
Producto final: debate y texto valorativo

1) Presentación de la SD y finalidad de la misma, 2) Explicación de los tipos de argumentos y 3) Sí señor oscuro: juego de cartas argumentativo - reflexión sobre los tipos de argumentos (pequeño grupo)

1) Puesta en común con el juego de la sesión anterior (diario de bitácora) 2) Explicación del género argumentativo debate a través de una infografía y 3 ) actividad de adjetivos y enigmas en grupo.

1) Actividades iniciales y visualización de algunos cortes de vídeos de Youtube acerca del tema del debate. (Estereotipos de hombres y de mujeres), 2) discusión en grupos sobre el tema principal y tesis que defiende la película a partir de los fragmentos y 3) puesta en común y argumentos que pueden utilizarse tanto a favor como en contra de los videos.

1) Preguntas / dudas y búsqueda de información entre los componentes de cada grupo en el debate. 2) División a la clase en los grupos y debate final evaluable acerca del siguiente tema: ¿Qué estereotipos existen en la sociedad con respecto al hombre y de mujer? y 3) texto argumentativo de opinión personal.

Tabla 1: Esquema de la SD que se llevó a cabo en secundaria

En estas sesiones, los contenidos trabajados mediante esta SD fueron los siguientes: 


\section{Contenido trabajado}

El estudio de los tipos de argumentos, contraargumentos y, al mismo tiempo, el uso de la argumentación con la finalidad de sintetizar una opinión.

Las características del género argumentativo debate y su puesta en práctica

La claridad expositiva y argumentativa tanto oral como escrita.

El uso de mecanismos retóricos con finalidad persuasiva.

Las características que ha de tener un ensayo argumentativo breve

La asimilación del concepto de estereotipo y el de prejuicio social como causa y consecuencia uno del otro.

La asimilación del prejuicio social como causa de discriminación (cultural, académica, social, etc.).

Tabla 2: Contenidos trabajados a partir de la secuencia didáctica anterior

\subsubsection{Implementación de las sesiones en secundaria}

La SD se implementó en tres cursos de $1 .^{\circ}$ de Bachillerato con un total de 60 estudiantes. La primera sesión se dedicó a la toma de contacto con la argumentación a partir del juego Sí señor oscuro (se explicará más adelante, § 4.1), cuyo leitmotiv principal consiste en que unos lacayos den buenas excusas a su jefe, el Señor oscuro, para justificar el fracaso de una misión.

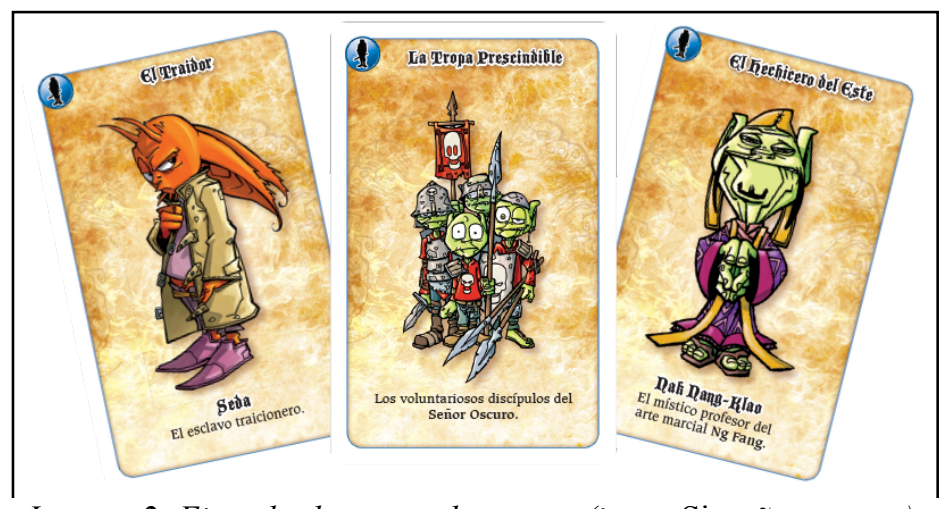

Imagen 2: Ejemplo de cartas de excusa (juego $\mathrm{Si}$, señor oscuro)

La segunda sesión se dedicó a la explicación de ciertos aspectos sobre la argumentación y de las reglas que se debían seguir para la tarea final. Al finalizarla, se repartió una tabla con 25 adjetivos de una terminación en los que debían señalar en grupo si referían a un hombre o a una mujer. 


\begin{tabular}{|c|c|c|}
\hline ADJETIVO & HOMBRE & MUJER \\
\hline \multicolumn{3}{|l|}{ Fuerte } \\
\hline \multicolumn{3}{|l|}{ Hábil } \\
\hline \multicolumn{3}{|l|}{ Grande } \\
\hline \multicolumn{3}{|l|}{ Independiente } \\
\hline \multicolumn{3}{|l|}{ Alegre } \\
\hline \multicolumn{3}{|l|}{ Débil } \\
\hline \multicolumn{3}{|l|}{ Fácil } \\
\hline \multicolumn{3}{|l|}{ Sutil } \\
\hline \multicolumn{3}{|l|}{ Flexible } \\
\hline \multicolumn{3}{|l|}{ Dificil } \\
\hline \multicolumn{3}{|l|}{ Amable } \\
\hline \multicolumn{3}{|l|}{ Deportista } \\
\hline \multicolumn{3}{|l|}{ Agradable } \\
\hline \multicolumn{3}{|l|}{ Insoportable } \\
\hline \multicolumn{3}{|l|}{ Inteligente } \\
\hline \multicolumn{3}{|l|}{ Triste } \\
\hline \multicolumn{3}{|l|}{ Profesional } \\
\hline \multicolumn{3}{|l|}{ Victimista } \\
\hline \multicolumn{3}{|l|}{ Mártir } \\
\hline \multicolumn{3}{|l|}{ Sensual } \\
\hline \multicolumn{3}{|l|}{ Paciente } \\
\hline \multicolumn{3}{|l|}{ Sensible } \\
\hline \multicolumn{3}{|l|}{ Inestable } \\
\hline \multicolumn{3}{|l|}{ Dependiente } \\
\hline Líder & & \\
\hline
\end{tabular}

Imagen 3: Tabla de adjetivos de una terminación

Como se aprecia, los adjetivos son de una única terminación y no se flexionan necesariamente en género. Por tanto, los alumnos en base a sus estereotipos elegían y, además, establecían una serie de topoi inconscientemente, dado que basaban sus argumentos en los estereotipos del tipo: una mujer es menos fuerte que un hombre (+mujer, - fuerte / +hombre, +fuerte). Para acabar, tuvieron otra hoja con los siguientes enigmas para debatir entre ellos 5 :

a) Un padre y su hijo viajan en coche y tienen un accidente grave. El padre muere y al hijo se lo llevan al hospital porque necesita una compleja operación de emergencia, para la que llaman a una eminencia médica. Pero cuando entra en el quirófano dice: «No puedo operarlo, es mi hijo».

b) Pérez tenía un hermano. El hermano de Pérez murió. Sin embargo, el hombre que murió nunca tuvo un hermano.

La tercera sesión se dedicó a aprender a contraargumentar una postura dada. Mediante varios anuncios y tráilers de películas sobre cuestiones relacionadas con los roles de género $^{6}$ se fue desentrañado cuáles eran las premisas que defendía cada muestra y cómo

\footnotetext{
${ }^{5}$ De esta forma, se trabajaba el estereotipo y su capacidad para detectarlo.

${ }^{6}$ Los enlaces al contenido visual son los siguientes:

- No soy un hombre fácil, tráiler de la película

https://www.youtube.com/watch?v=tPcFAklpV3k (entero)

- No soy un hombre fácil, escena de la película https://www.youtube.com/watch?v=wlIvMGMVCAY (entero)

- Mujer caminando por las calles de Nueva York https://www.youtube.com/watch?v=LQfmQbYFWCI (hasta el minuto)
} 
se podría llegar a contraargumentarlas. Finalmente, en esta sesión se repartieron al azar las posturas de cada grupo con respecto al debate final: feminista vs machista ${ }^{7}$. De esta manera, cada equipo puedo buscar información previa a la celebración del mismo. Finalmente, en la última sesión se celebró el debate. Antes de llevarlo a cabo, durante los primeros 15 minutos de la sesión los grupos terminaron de perfilar sus argumentos mediante búsquedas en internet. No obstante, la gran mayoría de los alumnos trajo información impresa (citas, recortes de prensa, estadísticas, etc.). Terminada esta fase de preparación, durante 25 minutos se efectuó el debate en torno al tema seleccionado desde el primer día: ¿Qué estereotipos existen en la sociedad con respecto al hombre y de mujer? Con el fin de evaluar todos los debates, se moderó cada uno de ellos in situ.
$1 .^{\circ} \mathbf{A}$
$1 .^{\circ} \mathbf{B}$
$10^{\circ} \mathrm{C}$ $\begin{array}{lll}2 \text { grupos de debate, es decir, un } & \begin{array}{l}6 \text { grupos de debate, es decir, } 3 \\ \text { debates simultáneos }\end{array} & \begin{array}{l}4 \text { grupos de debate, es decir, } 2 \\ \text { debates al mismo tiempo }\end{array}\end{array}$

Imagen 4: Número de debates por clase que se efectuó

De esta forma, creemos que podemos fomentar la expresión oral de carácter argumentativo donde se vea reflejada la opinión personal sobre el tema que se ha debatido, con esta opinión se aprecia qué orientación argumentativa tienen cada uno de los argumentos que se dan y, de esta forma, se puede resumir el carácter argumentativo de sus enunciados. Por esta razón, se ha considerado la posibilidad de adaptar toda esta secuencia didáctica para la enseñanza de ELE, dado que contiene una serie de actividades que, adaptadas, pueden contribuir al desarrollo tanto argumentativo como del conocimiento de la lengua de los estudiantes no nativos.

\section{4. ¿Qué se puede trabajar de argumentación en ELE?}

Se ha reseñado una propuesta que se llevó a cabo en el aula de secundaria y que se construyó con materiales propios y específicos para su implementación. No obstante, el objetivo de este artículo pretende adaptar esta secuencia para que se pueda aprovechar en la enseñanza de alumno no nativos de español.

Evidentemente, los objetivos de la secuencia que se implementó iban destinados a un alumnado que utilizaba como lengua vehicular el español y que lo que estaba aprendiendo eran los mecanismos correctos para desarrollar la competencia comunicativa de su lengua materna. Por el contrario, a nuestro alumnado de ELE se le garantizarán los materiales necesarios para afianzar su competencia argumentativa oral, teniendo en cuenta que lo que proponemos es una iniciación a la argumentación para convencer con la palabra y no solo para entender las palabras que oyen o aprenden. En definitiva, intentamos que los aprendientes, en este caso, aprendan la lengua mediante un mecanismo propio de esta: argumentar y razonar para convencer o ser convencidos.

\footnotetext{
-Youtuber criticando el concepto de micromachismo https://www.youtube.com/watch?v=iEcV4I7IXdA\&t=233s (primeros 2 minutos)

- Hombre caminando por las calles de Nueva York https://www.youtube.com/watch?v=7hHQYKp2E7I (primeros 35 segundos)

-Crítica a la concepción del hombre como género privilegiado https://www.youtube.com/watch? $v=p V \operatorname{sjn} A r K 1-\mathrm{M}$ (hasta el minuto 1:40)

${ }^{7}$ Se polarizaron tanto debido a que el alumnado necesitaba unas posturas bien definidas para argumentar mejor.
} 
En relación con nuestro propósito, la teoría de la argumentación en la lengua ya se ha utilizado en didáctica de ELE (en concreto, el aprovechamiento de los topoi); no obstante, en su gran mayoría desde el ámbito de la escritura y no de la oralidad (Garrido Rodríguez, 1999; Ibazeta, 2008; D’Agostino, 2011, etc. $)^{8}$. Por tanto, lo que proponemos a continuación, son una serie de actividades que potencian esa argumentación oral sustentadas en la teoría de la argumentación que se ha explicado anteriormente.

\subsection{Adaptación para el aula de ELE}

La secuencia anterior presenta un grupo de actividades que pueden ser muy útiles en el ámbito de ELE: ayuda a trabajar los adjetivos, contenidos argumentativos y la interacción sobre la base del enfoque comunicativo. Sin embargo, es necesario realizar una restructuración que permita focalizar el aprendizaje en el proceso y, de esta manera, permitir al alumnado no nativo adquirir todos los pasos necesarios para realizar la tarea final; que, en este caso también va a ser un debate (en un sentido laxo).

Por esta razón, se seguirán las premisas del enfoque por tareas, en el que un género discursivo concreto se desglosa en pasos pequeños para que los aprendientes asimilen su proceso de creación. A partir de estas premisas, se ha reestructurado la secuencia de la siguiente forma:

Número de sesiones

1. ${ }^{\circ}$ Sesión: toma de contacto con la argumentación

2. ${ }^{\circ}$ Sesión: ¿debate? ¿qué es debatir?

3. Sesión: preparación para el juego final

4. ${ }^{\circ}$ Sesión: juego final
Producto final: juego Sí, señor oscuro

1) Explicación de los tipos de argumentos, 2) visionado de videos que mínimamente argumenten una postura y 3) búsqueda de los argumentos que sustentan la opinión.

1) Puesta en común de los vídeos de la sesión anterior 2) aprendizaje del léxico y el vocabulario de los videos de la sesión anterior y 3) debate sobre las posturas que se defienden y por qué.

1) Lectura de la ficha de adjetivos de una terminación, 2) debate sobre qué significa cada término y 3) argumentación de qué postura se pude tomar con respecto al género de estos adjetivos: la orientación argumentativa.

1) Repaso de las sesiones anteriores, 2) Explicación sobre el juego al que se va a jugar para afianzar la argumentación y simular un debate y 3) juego final con una serie de posturas dadas, Sí, señor oscuro.

Tabla 3: rediseño de la secuencia didáctica aplicado a ELE

\footnotetext{
${ }^{8}$ El trabajo de la argumentación en la lengua puede afianzar el estudio de los distintos valores que una palabra tiene en contexto. Por ejemplo, para como preposición vs para como conector y su valor concesivo ( $\underline{\text { Para ser }}$ extranjero, Peter habla muy bien español; D’Agostino, 2011: 8). Es decir, se utiliza la orientación argumentativa de una partícula para explicar otros valores que no son los típicos de un habitual para (El regalo era para mí, entre otros valores).
} 
Como se puede comprobar en el rediseño de la secuencia didáctica implementada (tabla 3 ), se reutilizan materiales tales como la ficha de los adjetivos de una sola terminación (imagen 4) para realizar un doble trabajo: por un lado, entender por qué resulta problemático que ciertos adjetivos tengan una sola terminación que, dependiendo del contexto y la persona que los utilice, tendrán una interpretación u otra cuando nos refiramos a hombres o a mujeres. De esta manera, se promueve el trabajo de la orientación argumentativa $\mathrm{y}$; en este sentido, se trabaja inconscientemente con los topo $i^{9}$, por ejemplo, cuando decimos que el adjetivo fácil dependiendo del contexto tiene una interpretación para hombres y otra para mujeres queremos decir lo siguiente: a dos oraciones dadas como $2 a$ y $2 b$, la primera de ellas tendrá una lectura estereotipada y la segunda otra:

(2a) Es un hombre fácil con el que se puede hablar $(<+$ fácil, +tratable $>)$

(2b) Es una mujer fácil con la que se puede hablar (<+fácil, +sexual $>)$

$\mathrm{Y}$, por otro lado, se busca un tema polémico con el que los alumnos pueden debatir y utilizar todo lo aprendido sobre la argumentación. Se considera necesaria la contraposición de una interpretación y otra como eje vertebrador de un posible debate en el alumnado sobre los estereotipos que genera el sexo frente a los adjetivos, puesto que se enfrentan a la misma palabra con un mismo sentido, la facilidad, pero con una conclusión totalmente distinta dependiendo del topoi distinto en cada oración. En este sentido, fácil en $2 a$ remite a "Dicho de una persona: Accesible en el trato» (DLE, en línea) y fácil en $2 b$, si bien es cierto que conserva el sentido de accesible, «Dicho de una persona: Que se presta sin problemas a mantener relaciones sexuales» ( $D L E$, en línea), es totalmente distinto.

Del mismo modo, en la primera sesión se mantiene la clasificación de los tipos de argumentos, pero con cierta variación. Mientras que la teoría dirigida a los alumnos de secundaria, se basaba en lo que en un futuro podrían hacer en la prueba de selectividad (búsqueda de argumentos que refuerzan una tesis en un texto, selección y clasificación de los mismos, etc.), en un contexto de ELE se les expondrá a los aprendientes la existencia de los tipos de argumentos (imagen 5) y se intentará que a partir de esa clasificación vayan exponiendo ciertos argumentos y hablen entre ellos acerca de estos. También se recupera para esta sesión, la búsqueda de argumentos en videos, tráilers, etc. para sustentar lo aprendiendo con muestras distintas a las que han surgido en clase.

\footnotetext{
${ }^{9}$ Recordemos la teoría de la argumentación en la lengua definía topoi como una ley de paso general e incluso estereotipada (Fuentes y Alcaide, 2007: 36)
} 
Tipos de argumentos

\begin{abstract}
DATOS Y ESTADÍSTICAS $\rightarrow$ ya que un estudio, puesto que según los datos...
DE EXPERIENCIA PERSONAL $\rightarrow$ por esta razón yo..., puesto que / dado que en un momento de su vida..

CRITERIO SAPIENCIAL $\rightarrow$ porque en abril, aguas mil

VERDADES EVIDENTES $\rightarrow$ eso es imposible porque el agua hierve a $100^{\circ} \mathrm{C}$

ANALOGÍAS Y EJEMPLOS $\rightarrow$ esto fue así; no obstante / sin embargo esto de aquí...

DE AUTORIDAD $\rightarrow$ según esta persona / como dijo (persona que sabe de un tema)... esto es bueno, debido a / dado que está hecho / cantado / escrito, etc. por...
\end{abstract}

Imagen 5: muestra de cómo se pueden enseñar algunos argumentos

A partir de esta sesión, se podría fomentar otra en la que se estudiara el vocabulario utilizado en los videos expuestos $\mathrm{y}$, al mismo tiempo, se debatiese, mediante los argumentos aprendidos, el acuerdo o el desacuerdo con lo dicho en las muestras visionadas. Somos conscientes de que una parte de la argumentación se basa en la experiencia del hablante de saber según qué cosas sobre un tema, pero nosotros no buscamos que nuestros alumnos sobresalgan en todos los temas posibles que puedan sustentar un posible debate, sino que aprendan que existen ciertos mecanismos útiles que sirven para que ellos puedan, en un contexto medianamente argumentativo, sentirse competentes.

Si volvemos de nuevo a la secuencia implementada en secundaria (§ 3), se puede comprobar que el primer día se intentó explicar teoría (los tipos de argumentos) y, al mismo tiempo, esta se afianzó mediante una dinámica lúdica (un juego de cartas). Según muchos autores del ámbito de la didáctica de ELE, la inclusión del juego es óptima para conseguir alguno de los objetivos que tienen que asumir los aprendientes de español como segunda lengua (Sánchez Benítez 2012: 24-26; Báez Jaitkin 2016; Badia y Alemany, 2018). De hecho, desde la antropología se advierte que el juego, en su justa medida, puede ayudar a la comprensión del conocimiento y; sobre todo, constituye una parte importante de los seres humanos desde los inicios de nuestra especie y el establecimiento de nuestra propia cultura «El juego es más viejo que la cultura; pues, por mucho que estrechemos el concepto de ésta, presupone siempre una sociedad humana, y los animales no han esperado a que el hombre les enseñara a jugar» (Huizinga 2007: 7) ${ }^{10}$.

Por esta razón, la tarea final de esta secuencia continuará siendo un debate, pero conducido a partir del juego de cartas Sí, señor oscuro adaptado al ámbito de un aula de aprendientes de español. Mediante este, los contenidos que se van a poder trabajar son muy dispares y, al mismo tiempo, se consigue que el alumnado argumente en un

10 Sin obviar que, el juego solo es una herramienta más, no se debería sustentar en él todo el posible conocimiento que nuestros alumnos van a aprender sobre un tema. Como ayuda, en su justa medida, es bueno; pero no habría que convertirlo en el contenido de nuestras explicaciones, puesto que los contenidos son gramaticales, pragmáticos, sociolingüísticos, etc.; no lúdicos. 
contexto distendido, cercano y quizás más cómodo. Por lo tanto, el alumnado trabajará mediante este juego la argumentación a partir de la propia argumentación:
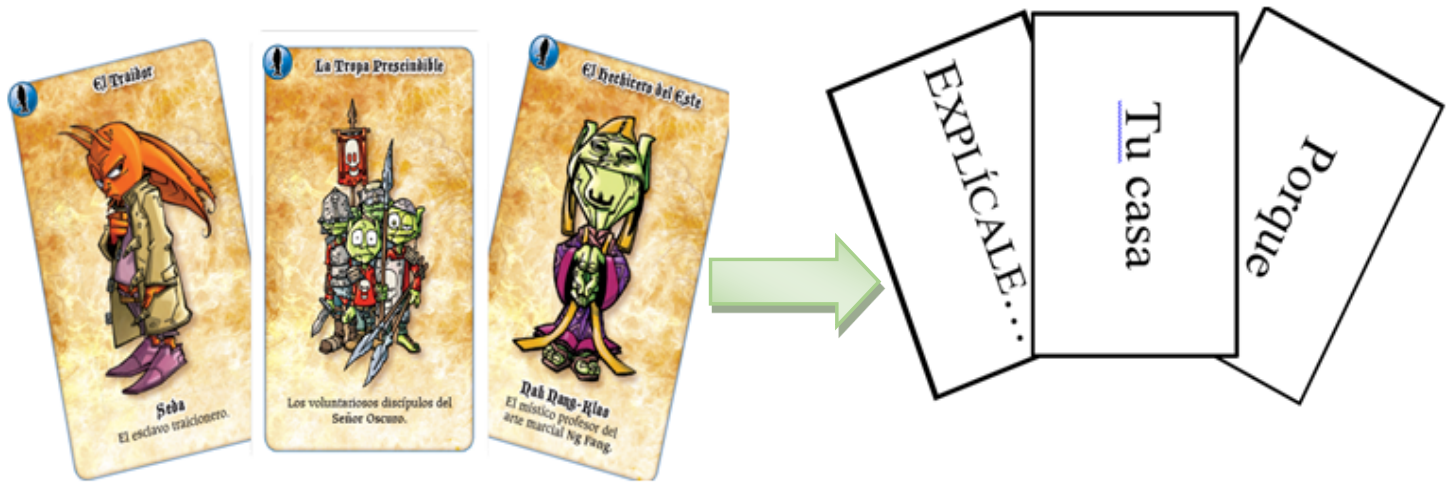

Imagen 6: Transformación a la didáctica de ELE del juego Sí, señor oscuro

Como se aprecia en la imagen 5 , se altera la dinámica del juego para que resulte útil su utilización en el aula de ELE. El juego base dispone de una dinámica sencilla: se designa a un Señor oscuro que manda sobre una serie de lacayos, este primero, pregunta a los otros integrantes cómo ha ido la misión que se les ha encomendado $\mathrm{y}$, acto seguido, los lacayos mediante una serie de cartas que poseen a priori tienen que excusarse sobre el porqué la misión ha fallo. Por ejemplo, en la imagen 5 se ve una carta que se titula El traidor, imagine el lector que el Señor oscuro le hace esta pregunta:

A (Señor oscuro): Mis lacayos, por qué no me habéis traído el libro que os pedí sobre El arte de la guerra. Tú, contéstame.

B (lacayo): Mi señor oscuro, si usted supiera lo que hemos tenido que pasar. No le hemos podido traer su libro porque había un traidor en nuestras filas ${ }^{11}$.

Es decir, el juego se resume en que unos lacayos argumentan (se excusan), en mayor o menor medida con cierta fundamentación, sobre por qué no han realizado el trabajo que les habían mandado.

El juego en el aula de secundaria no varió tanto de su propósito básico, si bien es cierto que el Señor oscuro no solo designaba las mejores excusas, sino que tenía que apuntar el tipo de argumento utilizado por sus lacayos. Si volvemos a la imagen 5, se comprueba que el cambio para ELE es sustancial, en vez de contar con 2 tipos de cartas, contamos con 3 :

${ }^{11}$ Como se comprueba, se utiliza una serie de argumentos y siempre el lacayo se ha de apoyar en la carta de excusa que tiene en su mazo. 


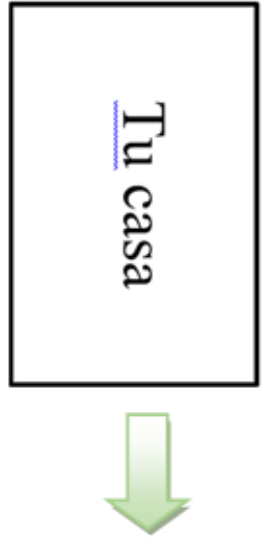

Cartas de excusa
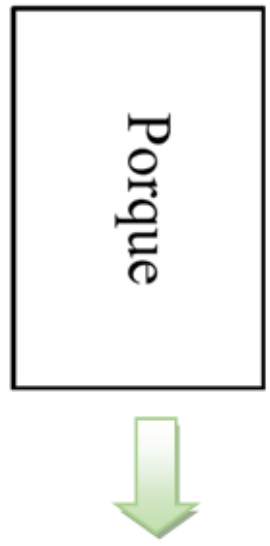

Conectores
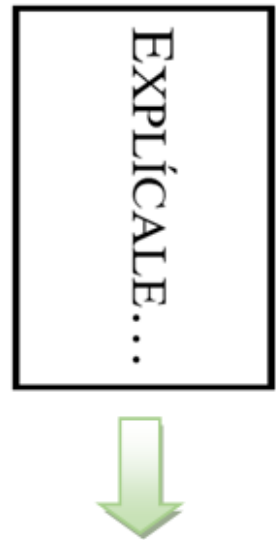

Cartas de acción

Imagen 7: Adaptación del juego a ELE

En este sentido, los aprendientes de español tienen que argumentar un hecho acerca de su casa mediante una orientación argumentativa dada, la causa (el conector porque). El tercer elemento que muestra la imagen 6 es una carta de acción, los lacayos que no sean el que se está excusando, pueden pedirle a su compañero que explique lo que ocurre con otra carta de excusa, como se muestra en el siguiente ejemplo inventado para aclarar nuestra explicación:

A (señor oscuro): ¿Por qué lacayos no me habéis traído mi libro de español como os pedí?

B (lacayo 1): Señor oscuro, usted no sabe lo que nos ha ocurrido. El libro no está en su casa porque no pudimos traerlo porque encontramos muchos problemas.

C (lacayo 2): Explícale [lanza la carta de acción más una de excusa de su mazo que pone «el perro»] lo que ocurrió con el perro que nos encontramos en el camino.

B: Señor oscuro, nos encontramos un perro que robó su libro.

De esta manera, como se puede apreciar en el ejemplo, se pueden trabajar una serie de contenidos:

\section{Contenido propuesto para trabajar}

Aprendizaje en contextos orales de conectores contraargumentativos como: no obstante, sin embargo, a pesar de, pero, etc.

Establecer reformulaciones en contextos argumentativos de interacción oral

Practicar los tipos de argumentos de autoridad, generalizaciones, experiencia personal, etc.

El uso de mecanismos con finalidad persuasiva para convencer al oyente.

Afianzar los contenidos que se han ido trabajando durante todo el curso (gramaticales, sociolingüísticos, pragmáticos, etc.)

El trabajo con las fórmulas de tratamiento (ustedeo y tuteo): Señor oscuro (usted), lacayos (tú)

La interacción oral

Tabla 4: Contenidos propuestos para trabajar en ELE

Otro aspecto que puede destacarse de esta actividad es la versatilidad que ofrece para el aula de ELE, ya que podemos modificar las cartas de las que disponen nuestro alumnado. Las cartas de excusa pueden ser muy diversas y adaptarse al vocabulario que estemos trabajando, así como los conectores y partículas. Por ejemplo, en vez de tener 
conectores, podemos disponer posturas / orientaciones argumentativas (a favor de o en contra de). De este modo, cuando tengan una carta como tu casa y una postura en contra, se pude conseguir una argumentación como la siguiente:

A: ¿Por qué lacayos no me habéis traído mi libro de español como os pedí?

B: Señor oscuro, no estoy de acuerdo con usted, si me permite. El libro ha estado aquí en su casa todo este tiempo, puede ser que se haya extraviado.

\section{Conclusiones}

La argumentación no es una categoría estanca de la lengua que se pueda estudiar desde un solo punto de vista, es una estrategia humana (Cuenca, 1995) sobre la que existen numerosas perspectivas de estudio y trabajo. Aunque en el ámbito de ELE se haya trabajado en mayor o menor medida desde una vertiente escrita (tipología textual, lingüística del texto, uso de conectores, etc.) la oral también es posible incorporarla a nuestro día a día como docentes. Sin ir más lejos, aunque suene tautológico, cada vez que un alumno opina sobre un tema de alguna manera argumenta o razona sobre ese tópico.

En este trabajo, se ha querido demostrar que los recursos empleados para el aula de secundaria pueden adaptarse para los estudiantes de ELE, aunque con una serie de matices y consideraciones que deben ser tenidas en cuenta en los procesos de adaptación de materiales. Por un lado, en el aula de secundaria, como se comprueba con la secuencia que se implementó, se trabaja la argumentación hacia un alumnado nativo en el idioma a partir de los contenidos curriculares. Esto es, la lengua meta es la de salida y por tanto los alumnos se identifican con ella y la asumen como propia.

Por otro lado, en el aula de ELE, los alumnos tienen diversas lenguas madre y se intentan aproximar a la misma lengua meta. Este hecho hace que los contenidos no se trabajen desde la asunción de una lengua nativa propia y común a todo el alumnado, sino que se alteren estos para conseguir la mayor comprensión de los aprendientes de español.

Como señalan el Instituto Cervantes y el Marco Común, la argumentación es tanto objetivo como vehículo para trabajar en el aula, ya que permite, no solo aprender las estructuras propias de los textos argumentativos, sino también desarrollar estructuras gramaticales, vocabulario o aprender géneros discursivos concretos como el anuncio (Badia, en prensa).

Asimismo, la argumentación permite dotar a los estudiantes no nativos de mecanismos para mejorar su razonamiento desde el español; ya que les da herramientas para desarrollar su pensamiento de un punto A hasta un punto B de forma coherente. Por ello, se consiguen tres movimientos: 1) entienda lo que se argumenta, 2) haga que su interlocutor comprenda por qué está argumentando y con qué fin y 3 ) convenza (o no) a su oyente.

Finalmente, cabe decir que esta aplicación tiene muchas más posibilidades, es cuestión del propio docente que la escoja llevar a cabo en una adaptación a sus clases y, con ello, seguir mejorando nuestros materiales para conseguir nuestro fin último: que nuestros alumnos aprendan español y, que, al mismo tiempo, sientan que son competentes en una 
estrategia tan humana como es razonar, debatir o argumentar con otros hablantes en un idioma que no es su lengua materna.

\section{Bibliografía}

ABASCAL, M. ${ }^{a}$ D. (2005). Retórica Clásica y Oralidad. Málaga: Analecta Malacitana.

Álvarez, G. y BEATRIZ GARCÍA, M. (2016). «Dificultades de estudiantes universitarios en la comprensión de textos argumentativos». Revista Educación y Humanismo, 19 (32), pp. 18-30. DOI: http://dx.doi.org/10.17081/eduhum.19.32.2529.

ADAM, J.M. (1992). Les textes: types et prototypes. París: Nathan.

AnsCombre, J.-C. (1995). «Semántica y léxico: Topoi, estereotipo y frases genéricas». Revista Española de Lingüistica, 25, pp. 297-310.

AnsCombre, J.C. y Ducrot, O. (1994). La argumentación en la lengua. Madrid: Gredos.

ARNOLD Morgan, J. (2006). «Los factores afectivos en el aprendizaje del español como lengua extranjera». Centro Virtual Cervantes.

BADIA Climent, S. (en prensa). «Nadie se queda atrás: la integración del alumnado de ELE en secundaria».

Badia Climent, S. y Alemany Martínez, A. (2018). "Game over: cartas, rol y comida». Foro de profesores de ELE, 14.

BÁEZ JAITKIN, C. E. (2016). "Gamificación, ¿reto o normalidad en el proceso de enseñanza aprendizaje?». Actas del XIII Encuentro Práctico de ELE del Instituto Cervantes de Nápoles, pp. 14-22.

CAMPS, A. y DolZ, J. (1995). «Introducción: Enseñar a argumentar: un desafío para la escuela actual». Comunicación, Lenguaje y Educación, 25, pp. 5-8.

CANAlE, M. (1995 [1983]). «De la competencia comunicativa a la pedagogía comunicativa de la lengua». Competencia comunicativa. Documentos básicos en la enseñanza de lenguas extranjeras. Madrid: Edelsa, pp. 63-82

CAtTAni, A. (2003). Los usos de la retórica. Madrid: Alianza.

Colombo, A. (1992). «Il testo argomentativo: presupposti pedagogici e modelli di analisi. I pro e i contro». En Colombo, A. Teoria e didattica dei testi argomentativi. Firenze: La Nuova Italia, pp. 59-84.

CONSEJO DE EUROPA (2018). Common European Framework of Reference for Languages: Learning, Teaching, Assement.

CuenCA, M. a J. (1995). «Mecanismos lingüísticos y discursivos de la argumentación». Comunicación, Lenguaje y Educación, 25, pp. 23-40.

D’Agostino, M. A. (2011). «Argumentación en la enseñanza de ELE avanzado: el para concesivo». Signos ELE, n. ${ }^{\circ} 5$.

DECRETO 87/2015, de 5 de junio, del Consell, por el que establece el currículo y desarrolla la ordenación general de la Educación Secundaria Obligatoria y del Bachillerato en la Comunitat Valenciana. Diari Oficial de la Comunitat Valenciana, 
València, España, 10 de junio de 2015.

Dolz, J. (1995). «Escribir textos argumentativos para mejorar su comprensión». Comunicación, Lenguaje y Educación, 25, pp. 65-77.

DOlZ, J. \& PASQUIER, A. (1996). Argumentar para convencer. Una secuencia didáctica de iniciación al texto argumentativo para el primer ciclo de la Educación. Navarra: Gobierno de Navarra. Departamento de Educación y Cultura.

- (2000). Escribo mi opinión. Una secuencia didáctica de iniciación a los textos de opinión para el tercer ciclo de Educación Primaria. Navarra: Gobierno de Navarra. Departamento de Educación y Cultura.

ESCANDELl VidAL, M. a V. (2014). Introducción a la pragmática. Barcelona: Ariel.

Felton K., M. (2007). «La discussió deliberativa: usar el discurs de classe per promoure el pensament crític», Tems d'Educació, 33, pp.183-201.

FISCHER, A. (2004). The logic of real arguments. UK: Cambridge University Press.

Fuentes RodríGuez, C. y AlCAIDE LARA R., E. (2007). La argumentación lingüística y sus medios de expresión. Madrid: Ariel/Libros.

GARRIDO RODRÍGUEZ, M. ${ }^{\mathrm{a}}$ del C. (1999). «Los conectores pragmáticos en la enseñanza del ELE: Argumentación y Relevancia». ASELE: Actas X Congreso.

GRIJELMO, Á. (2002). La seducción de las palabras: Madrid: Taurus.

- (2012). La información del silencio. Cómo se miente contando hechos verdaderos. Madrid: Taurus.

GRUPO GORGIAS (2013). Enseñar a debatir: guía para el profesorado. Murcia: Editum, Universidad de Murcia.

HuizINGA, J. (2007). Homo ludens. Barcelona: Editorial Alianza

IBAZETA, M. ${ }^{\mathrm{a}}$ V. (2008). «El uso de los textos argumentativos en la enseñanzaaprendizaje de ELE». Instituto Cervantes de Río de Janeiro.

InStituto Cervantes (2006). Plan curricular del Instituto Cervantes. Niveles de referencia para el español. Madrid: Instituto Cervantes- Biblioteca nueva.

JimÉNEZ AlEIXANDRE, P. (2009). Competencias en argumentación y uso de pruebas: 10 ideas clave. Barcelona: Graó.

Johnson, S. L. (2013). Ganar debates. New York - Amsterdam - Brussels: International Debate Education Association.

MAATI BEGHADID, H. (2013). «El enfoque comunicativo, una mejor guía para la práctica docente». Actas del IV taller «ELE e interculturalidad» Instituto Cervantes de Orán, pp. 112-120.

NunAN, D. (1989). El diseño de tareas para la clase comunicativa. Cambridge: Cambridge University Press.

Perelman, C. y Olbrechts-TyteCA, L. (1958). Traité de l'argumentation: La nouvelle rhétorique. Paris: Presses Universitaires de France. Traducción al español de Julia Sevilla Muñoz (1989). Tratado de la argumentación: La nueva retórica. Madrid: 
Gredos.

Plantin, C. (1998). La argumentación. Barcelona: Ariel Prácticum.

- (2012). La argumentación: historia, teorías, perspectivas. Traducción por Nora Isabel Muñoz. Buenos Aires: Biblos.

REAL DECRETO 1105/2014, de 26 de diciembre, por el que se establece el currículo básico de la Educación Secundaria Obligatoria y del Bachillerato. Boletín Oficial del Estado, Madrid, España, 3 de enero de 2015.

ReyzÁBAL, M. ${ }^{a}$ V. (2002). Didáctica de los discursos persuasivos: la publicidad y la propaganda. Madrid: La Muralla.

RodríGUEZ GONZALO, C. (2012). «L'argumentació a l'aula: entre el raonament científic i l’opinió social», Articles de Didàctica de la Llengua i de la Literatura (58), pp. 8-19.

SÁNCHEZ BENÍTEZ, G. (2010). «Las estrategias de aprendizaje a través del componente lúdico», MarcoELE: Revista de didáctica, ELE, 11.

SÁNCHEZ PRIETO, G. (2007). «El debate en el aula como herramienta de aprendizaje y evaluación». Jornadas Internacionales de Innovación Universitaria. Métodos docentes afines al E.E.E.S, celebradas en la Universidad Europea de Madrid el 12 y 13 de julio. Recuperado de http://abacus.universidadeuropea.es/bitstream/handle/11268/3294/S\%C3 $\%$ A1nchez $\% 20$ Prieto $\% 2 \mathrm{C} \% 20$ Guillermo.pdf?sequence $=$.

SCHOPENHAUER, A. (2018 [1864]). El arte de persuadir. Madrid: Editorial Verbum.

Toulmin, S. (1958). The uses of argument. Cambridge, England: Cambridge University Press.

VAN EEMEREN, F. H. y GROOTENDORST, R. (1992). Argumentation, communication and fallacies. A pragma-dialectical perspective. London and New York: Routledge Taylor and Francis Group.

Vilà Santasusana, M., Castellà, J. M. (2014). 10 ideas clave: Enseñar la competencia oral en clase. Aprender a hablar en público. Barcelona: Graó.

Vilà Santasusana, M., Castellà, J. M. y CASAs, M. (2018). Secretos para hablar bien en público: Guía práctica. Barcelona: Plataforma Editorial.

Weston, A. (2006). Las claves de la argumentación. Barcelona: Ariel.

ZAMUdiO DE MolinA, B. (2012). «Soportes de la argumentación. Lógica, dialéctica y retórica». En Investigación del Departamento de Humanidades y Ciencias Sociales 1 (1), 2250-8139, Buenos Aires: Universidad Nacional de La Matanza. 